\title{
Some results over thin-thick sets in cone locally convex spaces
}

\author{
Murat Cancan
}

Correspondence:

m_cencen@yahoo.com

Department of Mathematics,

Yuzuncu Yil University, 65080 Van, Turkey

\begin{abstract}
In this article, it is investigated some concepts of normed space like norming, thinthick sets in cone locally convex spaces. It is also worked some impacts of thick sets over the uniform quasi-boundedness principles in cone locally convex spaces such that weak*-bounded sets are strong ${ }^{*}$-bounded iff the space is a $B$ - $M$ space. It is demonstrated that these principles emerge under some weaker conditions with aim to thick sets. Moreover, it is displayed that the concept of thick is a duality invariant, that is, all suitable topologies for some cone locally convex space have the same thick sets.
\end{abstract}

Keywords: $B$ - M space, cone locally convex space, norming set, thin-thick set, uniform quasi-boundedness principle

\section{Introduction}

It has been described thin-thick sets for normed spaces in [1]. Therein, it is showed that there is an open mapping principle connected with thick sets in study of [1]. It is presented a wide survey for thick sets in $[2,3]$. In this studies it is unveiled some impacts of thick sets on certain operator spaces and also indicated that the boundedness and surjectivity results can be taken from this property.

$B(X)$ and $S(X)$ denote a closed unit ball and sphere of some normed space $X$, respectively. A set $U \subset X$ such that $\inf _{f \in S\left(X^{*}\right)} \sup _{x \in U}|f(x)| \geq \delta$, for some $\delta>0$, means norming for $X^{*}$, the continuous dual of $X$. A set $A$ means thick if for every representation of $A$ as the union of an increasing sequence of sets, $A=\bigcup_{n=1}^{\infty} A_{n},\left(A_{n}^{+}\right)$, there exists an index $m$ such that $A_{m}$ is norming. The set $A$ means thin if it is not thick. Moreover, a set $V \subset$ $X^{*}$ satisfying $\inf _{x \in S(X)} \sup _{f \in V}|f(x)| \geq \delta$, for some $\delta>0$, means norming for $X$ or $w^{*}$-norming. A set $B$ means $w^{*}$-thick if for every representation of $B$ as the union of an increasing sequence of sets, $B=\bigcup_{n=1}^{\infty} B_{n},\left(B_{n}^{+}\right)$, there exists an index $m$ such that $B_{m}$ is $w^{*}$-norming $[2,3]$. The definitions of the norming and $w^{*}$-norming sets are also qualified as follows $[3]$.

(L1) Let $X$ be a normed space, $A \subset X$ and $B \subset X^{*}$.

(i) $A$ is norming for $X^{*}$ iff $\overline{c o}( \pm A) \supseteq \delta B(X)$ for some $\delta>0$.

(ii) $B$ is norming for $X$ iff $\overline{c o}^{w^{*}}( \pm B) \supseteq \delta B\left(X^{*}\right)$ for some $\delta>0$. 
A subset $A$ of a normed linear space $X$ means to have the boundedness property if for every normed space $Y$, every family $\left(T_{\alpha}\right)$ in $L(X, Y)$, the space of all continuous linear operators from $X$ into $Y$, which is pointwise bounded on $A$, is bounded. It is evidenced in [2] a strong uniform boundedness principle valid on all Banach spaces for the thick sets. As an practice of this result it is given in [2] to a special case of the classical Banach-Steinhauss theorem; videlicet, suppose $\Gamma$ is a subset of $L(X, Y)$ and $B$ is a thick subset of $S(X)$. If $\Gamma(x)=\{T x: T \in \Gamma\}$ is a bounded subset of $Y$ for each $x \in B$, then $\Gamma$ is a bounded subset of $L(X, Y)$. The usual Banach-Steinhauss theorem is a special status of this result since every second category set is thick. Latterly, it is given a new property of thick sets by dint of weak integrability of Banach space valued measurable functions in [4]. It is also expressed thick sets by the agency of boundedness of vector measures, and clarify what case this concept is about the theory of barrelled spaces.

In this study it is aimed to find whether this type of important properties be given on uniform quasi-boundedness principles like equicontinuity of some family of operators between cone locally convex (clc, for short) spaces. Openly, the uniform boundedness principles in Banach spaces can tackle in two different forms in $c l c$-spaces:

(i) weak*-bounded sets are equicontinuous iff $X$ is barrelled,

(ii) weak*-bounded sets are strong*-bounded iff $X$ is a Banach-Mackey $(B-M$, for short) space $[5,6]$.

These two different forms coexist in quasi-barrelled spaces since strong"-bounded sets are equicontinuous in this status. It is represented that these principles happen under some weaker conditions through the medium of thick sets.

On the other hand, the fixed point, computer science and endpoint theory, concerning various single-valued and set-valued contractions, nonlinear contractions and asymptotic contractions in uniform, locally convex ( $l c$, for short) and metric spaces and in cone metric spaces, goes back to the original works by Banach [7]. Recently, renewed interest in this theory which is play a very important for many research areas as well as in function analysis arose also in different areas as applied mathematics.

Let $X$ be a real Banach space. A subset $K$ of $X$ means a closed convex pointed cone iff:

(i) $K$ is closed, nonempty, and $K \neq\{0\}$,

(ii) $r_{1}, r_{2} \in R, r_{1}, r_{2} \geq 0, x, y \in K \Rightarrow r_{1} x+r_{2} y \in K$,

(iii) $x \in K$ and $-x \in K \Rightarrow x=0$.

Given a cone $K \subset X$, we can define a partial ordering $\leq$ with respect to $K$ by $x \leq y$ iff $y-x \in K$. We write $x<y$ to indicate that $x \leq y$ but $x \neq y$, while $x<y$ will stand for $y$ $-x \in$ int $K$, int $K$ denotes the interior of $K$.

In most of the proofs in [8-11] cones are required to be restricted to a special case, namely to normal cones. The cone $K$ means normal if there is a constant number $k>$ 0 such that for all $x, y \in X, 0 \leq x \leq y$ implies $\|x\| \leq k\|y\|$. The least positive number satisfying the above inequality means the normal constant of $K$. 
(T1) Let $X$ be a vector space over $R$ and let $E$ be an ordered Banach space with cone $K$ where $I$ is a index set:

(i) The family $Q=\left\{q_{\alpha}: X \rightarrow E, \alpha \in I\right\}$ means to be a $Q$-family of cone seminorms on $X$ (Q-family, for short) if the following three conditions hold:

(i*) $\forall \alpha \in I, \forall x \in X,\left\{0 \leqslant q_{\alpha}(x) \wedge x=0 \Rightarrow q_{\alpha}(x)=0\right\}$;

(ii*) $\forall \alpha \in I, \forall \lambda \in R, \forall x \in X,\left\{q_{\alpha}(\lambda x)=|\lambda| q_{\alpha}(x)\right\}$; and

(iii:) $\forall \alpha \in I, \forall x, y \in X,\left\{q_{\alpha}(x+y) \leqslant q_{\alpha}(x)+q_{\alpha}(y)\right\}$.

(ii) If $Q$ is a $Q$-family, then the pair $(X, Q)$ means a $c l c$ space.

(iii) A $Q$-family $Q$ means to be separating if:

(i*) $\forall x \in X,\left\{x \neq 0 \Rightarrow \exists \alpha \in I,\left\{0 \prec q_{\alpha}(x)\right\}\right\}$.

(iv) If a $Q$-family $Q$ is separating, then the pair $(X, Q)$ means a Hausdorff $c l c$-space (hclc space, for short) [12].

In this study, it is investigated thin-thick sets of $c l c$ spaces endowed with a important place in the fixed point with endpoint theory and being a natural generalization of $l c$ spaces.

\section{Extensions and some important results}

Definition 2.1. For a subset $A$ of a $c l c$-space $X(T 1)$, the polar of $A$, written $\dot{A}$, is $\{f \in$ $X^{*}:|f(x)| \leqslant 1$, for all $\left.x \in A\right\}$. In dual sense, for $A \subset X^{*}$, $\dot{A}=\{x \in X:|f(x)| \preccurlyeq 1$, for all $f \in A\}$. For all $A, \dot{A}$ is absolutely convex and closed in any suitable topology for $X^{*}$ and $\ddot{A}$ is the absolutely convex closure of $A$. For the definition of the strong-topology (strong"-topology) $\beta\left(X, X^{*}\right)\left(\beta\left(X^{*}, X\right)\right)$ of $X\left(X^{*}\right)$, it can be refered to [5,6]. If $X$ is a normed space, $\beta\left(X^{*}, X\right)$ is (equivalent to) the norm topology in $X^{*}$ on the strength of $[5,6]$. However, $\beta\left(X, X^{*}\right)$ need not be the norm topology for $X$ even if $X$ is a normed space where the Mackey ( $M$, for short)-topology $\tau\left(X, X^{*}\right)$ is the norm topology for $X$ in this status. Note that $\tau\left(X, X^{*}\right)$ is the strongest $c l c$-topology (for $X)$ suitable with the dual pair $\left(X, X^{*}\right)$. Let $T$ be a $c l c$-topology for $X$ and $S$ be a subset of $(X, T) *$. Then $S$ is equicontinuous iff $\dot{S}$ is a neighborhood of 0 . In dual sense, $\dot{U}$ is equicontinuous whenever $U$ is a neighborhood of 0 . In a seminormed space $X$, a set $S$ $\subset X^{*}$ is equicontinuous iff it is norm bounded. In this case, the equicontinuity is a generalization of the uniform boundedness. A barrelled space is a $h c l c$-space in which every barrel is a neighborhood of 0 . Remember that a barrel is an absolutely convex absorbing closed set. The initial perspective of barrelled spaces was in reply to the question of finding an internal qualification of spaces where the uniform boundedness principle holds. The reply is given by the following characterization by virtue of $[5,6]$.

Theorem 2.1. Let $X$ be a $c l c$-space. Then the following three conditions are equivalent:

(i) For any $c l c$-space $Y$, every pointwise bounded family $S$ of continuous linear maps from $X$ to $Y$ is equicontinuous.

(ii) Every weak ${ }^{*}$-bounded subset of $X^{*}$ is equicontinuous.

(iii) $X$ is barrelled.

It is an important property of a barrelled space that its topology is the strong-topology on the strength of [6]. A hclc-space means quasi-barrelled if every bornivorous barrel is a neighborhood of 0 . Nonforget that a bornivorous is a set which absorbs all 
bounded sets. The natural embedding of a hclc-space $X$ into $X^{* *}=\left[X^{*}, \beta\left(X^{*}, X\right)\right]^{*}$ is the map $\hat{x}$ where $\hat{x}(f)=f(x)$ for $f \in X^{*}$. It is a homeomorphism iff $\mathrm{X}$ is quasi-barrelled. A $h c l c$-space is $X$ quasi-barrelled iff all strong"-bounded sets are equicontinuous. A hclcspace $X$ means semireflexive if $X=X^{* *}$, that is, the natural embedding is onto $X^{* *}$. It means reflexive if the natural embedding is a homeomorphism of $X$ onto $X^{* * *}$. Thus reflexive means semireflexive and quasi-barrelled. A $h c l c$-space means a $B-M$ space if all its bounded sets are strongly bounded.

Now we proceed by the extended definition of norming sets in $c l c$-spaces.

Definition 2.2. Let $X$ be a vector space and $T$ be a $c l c$-topology for $X$. A set $A \subset X$ means $T$-norming for $(X, T)^{*}$ whenever $\overline{c o}^{T}( \pm A)$, the absolutely convex closure of $A$, includes a $T$-neighborhood of 0 .

Remark 2.1. Let $T$ be normable, that is, $(X, T)$ be a normed space. Then $T$ is characterized by balls, and so finding some $\delta>0$ such that $\overline{c o}^{T}( \pm A) \supseteq \delta B(X)$ is a sufficient condition for the definition of a norming set. Moreover, let $X$ be a dual space of a normed space $Y$ with the $w^{*}$-topology. Then, by the definition, a set $A \subset X=Y^{*}$ is $w^{*}$ norming for $\left(X, w^{*}\right)^{*}=Y$ iff $\overline{c o} w^{w^{*}}( \pm A)$ includes a $w^{*}$-neighborhood $U$ of 0 . But, $U$ is also a norm-neighborhood of 0 in $X$ and hence $\overline{c o}^{w^{*}}( \pm A) \supseteq \delta B\left(Y^{*}\right)$ for some $\delta>0$. Thus, Definition 2.2 includes earlier definitions of both norming and $w^{*}$-norming sets by means of $(L 1)$. The other definitions of [13] like strong and strong"-norming sets are derived from Definition 2.2 taking $T$ as $M$, strong and strong"-topology, respectively.

Definition 2.3. Let $X$ be a vector space and $T$ be a $c l c$-topology for $X$. A set $A \subset X$ means $T$-thick if for every representation of $A$ as the union of an increasing sequence of sets, $A=\bigcup_{n=1}^{\infty} A_{n},\left(A_{n}^{+}\right)$, there exists an index $m$ such that $A_{m}$ is $T$-norming for $(X, T)^{*}$. The set $A$ means $T$-thin if it is not $T$-thick.

We see to benefit using only the words norming, thin-thick whenever $T$ is clear from the context.

It can see easily as in $[2,5]$ that a set in a $c l c$-space which is of the second category is thick.

Theorem 2.2. For a $c l c$-space $X$, all suitable topologies for $X$ have the same norming sets. Hence, thick concept is a duality invariant.

Proof. This comes from the fact that the closures of convex sets in all suitable topologies are the same, that is, a closed convex set is a duality invariant. Also it is possible to express it on the strength of both [14] and $[5,6]$. Hence with this result it is subjointed an argument to the list of duality invariants as presented in $[5,6]$. It is well known that the strong-topology $\beta\left(X, X^{*}\right)$ may not be suitable even if $X$ is a normed space.

There is some examples of normed spaces as $[5,6]$ such that the strong-topology is strictly larger than the $M$-topology.

Now, we give an example of a set which is strong-norming but is not $M$-norming.

Example 2.1. Let $X=C_{00}[-1,1]$, the set of real continuous functions on the interval $[-1,1]$, each of which vanishes on a neighborhood of 0 , with the sup norm $\|\cdot\|_{\infty}$. For each $n \in N, f_{n}(x)=n x(1 / n)$ is a continuous linear functional on $X$ since $\left|f_{n}(x)\right| \leqslant \|$ $x \|_{\infty}$. Let $A=\{x \in X:|x(1 / n)| \leqslant 1 / n\}$ and $B=\left\{f_{n}\right\}_{n=1}^{\infty} \cup\{0\}$. Then 
$A=\dot{B}=\left\{x:\left|f_{n}(x)\right|=|n x(1 / n)| \preccurlyeq 1\right\}$. The set $B$ is $w^{*}$-bounded in $X^{*}$. Since the set of $w^{*}$-bounded sets is the polar family for the strong-topology like [14,15], $A$ is a strongneighborhood of 0 and hence is strong-norming. However, $A$ is not $M$-norming. Let us prove this. Since the $M$-topology $\tau\left(X, X^{*}\right)$ is the strongest suitable topology for $X$, $\overline{c o}^{\tau\left(X, X^{*}\right)}( \pm A)=A$. But $A$ is not a $M$-neighborhood of 0 . To prove this assertion, let us assume that $A$ is a $\tau\left(X, X^{*}\right)$-neighborhood of 0 . In a normed space, $M$-topology is the norm topology, and so $A$ is a $\|\cdot\|_{\infty}$-neighborhood of 0 . Hence, $A$ must contain $\delta B(X)$ for some $\delta>0$. But this is not the case. Indeed, consider the function $x:[-1,1] \rightarrow R$,

$$
x(t)= \begin{cases}\delta / 2 & \text { if } t=1 / n, n=1,2,3 \\ 0 & \text { if otherwise. }\end{cases}
$$

Then $x \in \delta B(X)$ while $x \notin A$. This proves the claim. For dual spaces, $w^{*}$-topology is not suitable with the strong*-topology, in general. Hence, we cannot expect $w^{*}$-thick sets to be strong"-thick up to semireflexivity.

Theorem 2.3. In a semireflexive $h c l c$-space $X, w^{*}$-thick sets are strong*-thick.

Proof. It is expressed from $[5,6]$ that a $h c l c$-space $X$ is semireflexive iff the $w^{*}$-topology is suitable with the strong"-topology. Hence the result follows from Theorem 2.2.

Remark 2.2. (i) Theorem 2.3 is a generalization of a general conjecture where for a reflexive Banach space $X, w^{*}$-thick sets are (norm) thick in $X^{*}$. This comes from the facts that $\left(i^{*}\right)$ the norm topology of the dual is equivalent to the strong*-topology and $\left(i i^{*}\right)$ a Banach space is reflexive iff it is semireflexive from [5,6]. (ii) For a $c l c$-space $X$, the uniform boundedness principles for a Banach space appear in two different forms: $\left(i^{*}\right) w^{*}$-bounded sets are equicontinuous iff $X$ is barrelled; $\left(i i^{*}\right) w^{*}$-bounded sets are strong"-bounded iff $X$ is a $B-M$ space. These two different forms coexist in quasi-barrelled spaces since strong*-bounded sets are equicontinuous in this case. Remember that a quasi-barrelled $B-M$ space is a barrelled space.

Now, let us inspect some impacts of thick sets using these principles. The following theorems extend the main results of $[2,5]$ in these respects.

Theorem 2.4. Let $(X, T)$ be a $B-M$ space and $A$ be a thick subset of $X$. Suppose that $B \subset X^{*}$ is pointwise bounded on $A$, i.e., the set $B(x)=\{f(x): f \in B\}$ is bounded in $R$ for each $x \in A$. Then $B$ is strong"-bounded.

Proof. Since $X$ is a $B-M$ space, so also is $X^{*}$. Hence, if we show that $B$ is a $w^{*}$ bounded then we have that $B$ is also strong"-bounded. Let $A_{n}=\{x \in A:|f(x)| \leqslant n$, for each $f$ in $B\}$, so that each $A_{n}=[(1 / n) B]^{\circ} \cap A$ and $A=\bigcup_{n=1}^{\infty} A_{n}\left(A_{n}^{+}\right)$. Since $A$ is thick, there is a positive integer $m$ such that $A_{m}$ is norming for $(X, T)^{*}$. Then $\overline{c o}^{T}\left( \pm A_{m}\right)$ includes a neighborhood of 0 . But $\overline{c o}^{T}\left( \pm A_{m}\right) \subseteq[(1 / m) B]^{\bullet}$ since a polar is an absolutely convex and closed set. This implies $[(1 / m) B]^{*}$ is a neighborhood of 0 in $(X, T)$ and hence $(1 / m) B$ is an equicontinuous set in $(X, T)^{*}$. This implies $B$ is a weak*-bounded set in $(X, T)^{*}$.

Definition 2.4. A subset $A$ of a $c l c$-space $X$ means to have the equicontinuity property if for every $c l c$-space $Y$, every family $\left(T_{\alpha}\right) \subset L(X, Y)$ which is pointwise bounded on $A$ is equicontinuous.

Theorem 2.5. Let $A$ be a thick subset of a $c l c$-space $X$. Then the following three conditions are equivalent: (i) $A$ has the equicontinuity property for $c l c$-spaces; (ii) $A$ has the equicontinuity property for $R$; (iii) $X$ is barrelled. 
Proof. $(i) \Rightarrow(i i)$ is obvious. For $(i i) \Rightarrow(i i i)$, let $A$ have the equicontinuity property for $R$. This means that a set $B \subset X^{*}$ which is pointwise bounded on $A$ is equicontinuous. Suppose $U \subset X$ is a barrel and $f \in \dot{U}$. Pick some $x \in X$, and let $\varepsilon>0$ so that $\varepsilon x \in U$. Then $|f(x)|=(1 / \varepsilon)|f(\varepsilon x)| \leqslant 1 / \varepsilon$. Hence $\dot{U}$ is $w^{*}$-bounded since $x$ is arbitrary. This implies, clearly, that $\dot{U}$ is pointwise bounded on $A$, and hence equicontinuous by the hypothesis. Moreover, $U=\ddot{U}$ because $U$ is absolutely convex. Thus, it is a neighborhood of 0 since it is the polar of an equicontinuous set, whence $X$ is barrelled. For (iii) $\Rightarrow(i)$, let $Y$ be a $c l c$-space and $S$ be any subset of $L(X, Y)$ such that $S(x)=\{T(x): T \in L$ $(X, Y)\}$ is bounded in $Y$ for each $x \in A$. In a similar way to the proof of Theorem 2.4 shows that $S$ is pointwise bounded on the whole space, and hence equicontinuous from Theorem 2.1.

\section{Competing interests}

The author declares that they have no competing interests.

Received: 3 February 2011 Accepted: 5 September 2011 Published: 5 September 2011

\section{References}

1. Kadets, MI, Fonf, VP: Two theorems on the massiveness of the boundary in a reflexive Banach space. Funct Anal Appl. 17, 227-228 (1983)

2. Nygaard, O: Approximation boundedness surjectivity. Dr Scient Thesis. University of Bergen (2001)

3. Nygaard, O: Boundedness and surjectivity in normed spaces. Int J Math Math Sci. 32, 149-165 (2002). doi:10.1155/ S0161171202011596

4. Abrahamsena, TA, Nygaard, O, Poldvere, M: On weak integrability and boundedness in Banach spaces. J Math Anal Appl. 314(1), 67-74 (2006)

5. Yilmaz, Y: Thin and thick sets in locally convex spaces. Nonlinear Anal. 67, 1440-1444 (2007). doi:10.1016/j. na.2006.07.028

6. Wilansky, A: Modern Methods in Topological Vector Spaces. McGraw-Hill, New York (1978)

7. Banach, S: Sur les opérations dans les ensembles abstraits et leurs applications aux équations intégrales. Fund Math. 3 , 133-181 (1922)

8. Abbas, M, Rhoades, BE: Fixed and periodic point results in cone metric space. Appl Math Lett. 22, 511-515 (2009). doi:10.1016/j.aml.2008.07.001

9. Abbas, M, Jungck, G: Common fixed point results for noncommuting mappings without continuity in cone metric spaces. J Math Anal Appl. 341, 416-420 (2008). doi:10.1016/j.jmaa.2007.09.070

10. Abdeljawad, T: Completion of cone metric spaces. Hacettepe J Math Stat. 39(1), 67-74 (2010)

11. Long-Guang, H, Xian, Z: Cone metric spaces and fixed point theorems of contractive mappings. J Math Anal Appl. 332 1468-1476 (2007). doi:10.1016/j.jmaa.2005.03.087

12. Wlodarczyk, K, Plebaniak, R, Dolinski, M: Cone uniform, cone locally convex and cone metric spaces, endpoints, setvalued dynamic systems and quasi-asymptotic contractions. Nonlinear Anal. 71, 5022-5031 (2009). doi:10.1016/j. na.2009.03.076

13. Mackey, GW: On infinite-dimensional linear spaces. Trans Am Math Soc. 57, 155-207 (1945). doi:10.1090/S0002-99471945-0012204-1

14. Köthe, G: Topological Vector Spaces I. Springer. (1969)

15. Nygaard, O: A strong uniform boundedness principle in Banach spaces. Proc Am Math Soc. 129, 861-863 (2001). doi:10.1090/S0002-9939-00-05607-0

doi:10.1186/1687-1812-2011-48

Cite this article as: Cancan: Some results over thin-thick sets in cone locally convex spaces. Fixed Point Theory and Applications 2011 2011:48. 